\title{
ANÁLISIS COMPARATIVO ENTRE RAZAS DE PALOMOS BUCHONES EN BASE A CARACTERÍSTICAS MORFOLÓGICAS
}

\author{
Comparative Analysis of Morphological Characteristics in Pouter \\ Pigeon Breeds
}

\author{
Pere-Miquel Parés i Casanova ${ }^{1}$
}

\section{RESUMIEN}

Se estudió 18 razas de palomos buchones, españolas e iberoamericanas, a partir de la evaluación de 28 características morfológicas en base al principio de parsimonia usando el método de Fitch para el análisis cualitativo, y se generó un dendrograma de clusters por el método de varianza mínima entre los grupos de Ward para el análisis cuantitativo. Se detecta una cierta lógica en los árboles obtenidos, pero las relaciones no se manifiestan con claridad. El Índice de Consistencia fue de 0.38 y el Índice de Retención de 0.412 , lo que indica un ajuste medio entre los datos y el cladograma. Los resultados indican que algunas de las características morfológicas podrían no ser informativas, o bien, que datos complementarios, como los relacionados con el comportamiento, podrían proporcionar una mejor información para la construcción de un árbol de clasificación lógica para las razas de buche.

Palabras clave: cladística, etnología, morfología, buchón ladrón, variedad

\section{AbSTRACT}

Relationships among 18 Spanish and Iberoamerican pouter pigeon breeds were studied using data from 28 morphological characteristics based on the parsimony principle by the Fitch method for the qualitative analysis. A cluster dendogram was generated by the minimum variance between Ward groups for the quantitative analysis. Some logic in the trees was found but no clear relationships between breeds were observed. The value of Consistency Index was 0.38 and for the Retention Index was 0.416 , indicating a medium fit between data and cladogram. The results showed that some of the morphological characteristics could not be informative or that complementary traits such behavioral ones would give better information in the construction of a logical classification tree for pouter pigeon breeds.

Key words: cladistic, ethnology, morphology, thief pouter, variety

\footnotetext{
${ }^{1}$ Depto. Producció Animal, Ciencia i Salut Animal (ETSEA), Universitat de Lleida, Catalunya, España. E-mail : peremiquelp@prodan.udl.cat
} 


\section{INTRODUCCIÓN}

Desde tiempos remotos se practica en España lo que hoy se llama "Columbicultura Deportiva". En Andalucía y en el Levante español se tiene el mayor número de aficionados a la columbicultura, y ello se refleja en el número de razas locales, mayoritariamente buchonas, parte de las cuales han originado, a su vez, razas en Iberoamérica.

Todas las razas de palomas domésticas del mundo descienden de la paloma bravía (PAB, Columba livia Gmelin, 1789) (del Hoyo et al., 1997). La PAB es natural de Eurasia y del norte de África, posee un plumaje gris azulado, más pálido en el dorso, con verde y violeta lustroso en los lados del cue1lo, obispillo blancuzco, dos anchas franjas alares y blanco debajo de las alas, y con franja terminal negra en la cola. Su tamaño es el eumétrico en las palomas (250-300 g) y sus proporciones son medio-líneas (Sotillo y Serrano, 1985).

Se considera que la paloma buchona fue traída a España por los árabes en la época de la Edad Media, a raíz de su invasión en el año 711. Las razas españolas más antiguas parecen ser el Rafeño que data del siglo XI y el Marchenero del siglo XVI. El Quebrado Murciano se describe igualmente como una raza muy antigua (Schille, 2005).

Las características comunes de los palomos buchones son el buche desarrollado, patas no emplumadas, variedad de color en la mayoría de los casos, gran capacidad de vuelo, así como un carácter de trabajo basado en la búsqueda de la paloma perdida o desemparejada, en la seducción y captación y en el instinto de conservación (Sotillo y Serrano, 1985). El palomo buchón debe cumplir con los requisitos fenotípicos establecidos en sus respectivos patrones, y por otro lado, el trapío y la conquista. Aún así, las diferentes razas suelen presentar marcadas diferencias morfológicas. Suelen ser aves grandes con un tamaño desmesurado del bu- che, que al hincharse adquiere grandes proporciones, lo que les da el nombre a estos palomos. Tanto los machos como las hembras pueden «hincharse», aunque los primeros alcanzan mayor perímetro. La aparición de una hembra induce al pavoneo de los machos, que son cortejadores impenitentes, recibiendo por ello el apodo de «buchones ladrones». El concepto, o calificativo erróneo, aplicado a la paloma buchona, de «ladrona», puede obedecer a que estos palomas, llevados por su instinto de captación y de seducción, pueden llegar a apoderarse ilícitamente de palomos, pero es un término que debe evitarse en el vocabulario etnológico.

Los estudios comparativos entre razas de palomas son escasos; sin embargo, se destaca el trabajo de Barba et al. (1998) sobre el Buchón Jienense. En este trabajo se presenta un estudio de las relaciones existentes entre diferentes razas de palomos buchones españoles e iberoamericanos, realizado a partir del análisis cualitativo y cuantitativo de la información generada del estudio de sus características morfológicas, con el objetivo de hacer una clasificación sistemática de las diferentes razas en grupos afines, en base a semejanzas morfológicas.

\section{Materiales y Métodos}

Se realizó un estudio morfológico comparativo con 18 razas de palomos buchones. De estas, 15 razas fueron españolas: Buchón Balear (en denominación vernácula catalana "Gavatxut", GAV), Buchón Canario (CAN), Buchón Colillano (COL), Buchón Gaditano (también denominado "Isleño" o "Jerezano", GAD), Buchón Granadino (GRA), Buchón Jienense (JIE), Buchón Laudino Sevillano (SEV), Buchón Marchenero, también denominado Colitejo (MAC), Buchón Marteño (MAT), Buchón Moroncelo (MOR), Buchón Quebrado Murciano (QUE), Buchón Valenciano (VAL), Buchón Veleño (mal denominado "Castellano", VEL), Nuevo Buchón Valenciano (NVA) y Rafeño (RAF); y tres 
razas iberoamericanas: Buchón Alteño (ALT), Buchón Valenciano Argentino (ARG) y Colguero Argentino (CAR).

La PAB se utilizó como ancestro de las palomas buchonas y sus datos fueron recabados (Peterson et al., 1980; Gómez de Silva et al., 2005).

Se consideró 28 características morfológicas cualitativas con valor etnológico, tanto regionales como globales (Roelfzema, 2007). El estado de cada característica para cada raza se estableció a partir de las descripciones dadas por el patrón racial (en las razas que lo poseen), de las descripciones proporcionadas por Levy (1965), Mackrott (1997) y Schille (2005) y, en caso de ausencia, se usó las informaciones proporcionadas por los propios criadores. Los números para cada estado se asignaron de manera arbitraria, no implicando ningún peso específico. El número de estado para cada característica se estableció por el número de clases fenotípicas distinguibles, variando de 2 a 4, evitándose los estados únicos (Cuadro 1).

Las características continuas se dividieron en clases, cada uno representando uno de los estados de las características de la matriz de datos (estados 1, 2, 3, 4). La matriz original de las similitudes morfológicas se muestra en el Cuadro 2.

El estudio se basó en el principio de parsimonia usando el método de Fitch para el análisis cualitativo. Su generación asume que el árbol generado (cladrograma) es aquel que requiere el menor número posible de pasos o transiciones del estado de la característica, sumados a través de todas sus ramas. En este método, las características son reversibles y no están ordenadas, de manera que cualquier cambio tiene el mismo coste. La rotación del árbol es igualmente arbitraria. El algortimo heurístico usado fue el SPR ("Subtree Pruning and Regrafting") (Kitching et al., 1998) y se calcularon los límites de confianza de la topología mediante un análisis bootstrap (porcentaje de réplicas que suporta un grupo) a través de un centenar de réplicas.
Para evaluar el ajuste de los datos al árbol se eligió el Índice de Consistencia (IC) y el Índice de Retención (IR) (Naylor y Kraus, 1995). El IC es un estimado del grado de homoplasias esperadas en el árbol donde valores iguales a 1 indican ausencia de homoplasia. El IC para las características se define como: $\mathrm{IC}=\mathrm{M} / \mathrm{S}$, siendo "M" el número de estados menos uno de la característica y "S" el número de cambios de estado observados. El IR es un estimado de la cantidad de sinapomorfias esperadas en el árbol (Farris, 1989) y se define como RI = $(\mathrm{G}-\mathrm{S})$ / $(\mathrm{G}-\mathrm{M})$ donde «G» es el número de pasos necesarios en el caso del peor camino evolutivo posible, o sea, el número de pasos para la característica en un cladograma sin resolver, y para una característica determinada se elige el que sea numéricamente menor. El IR es alto cuando los cambios de estado ocurren predominantemente en los nodos internos y bajo cuando los cambios están concentrados en ramas pertenecientes a taxones terminales. Este índice tiene la ventaja de que no es sensible a las características no informativas, autapomorfías o sinapomorfías.

Los árboles de consenso son un tipo de árbol que pueden ser considerados como árboles derivados. Se construyen a partir de otros árboles y resumen a un conjunto de árboles. Una aplicación básica frecuente es la de reunir en un solo árbol la presencia de varios árboles resultado de ser los más parsimoniosos en el análisis. Por ello, se obtuvo un árbol de consenso basado en la regla del consenso de regla-estricta.

Para el análisis cuantitativo se obtuvo, en primer lugar, las distancias euclideanas y con esta matriz se generó un dendrograma de clusters por el método de varianza mínima entre los grupos de Ward. Con este método se minimiza la suma de los cuadrados de los residuos de cada dos hipotéticos clusters que pueden ser formados en cada paso, intentando que los conglomerados sean lo más homogéneos posible; y las desviaciones son las distancias de los puntos a las 
medias de los conglomerados a los que pertenecen. La generación de los árboles y el cálculo de las distancias se realizaron mediante el paquete PAST - "Paleontological Statistics Software Package for Education and Data Analysis" (Hammer et al., 2001).

\section{Resultados}

El cladograma resultante de la aplicación del método de parsimonia de Fitch en las características morfológicas se muestra en la Fig. 1. La parsimonia evaluó 4,300 árboles. Los valores en el árbol indican el nombre de réplicas (intervalo de confianza) del análisis bootstrap. La parsimonia necesitó 128 saltos (longitud total del árbol) para reordenar las características y obtener el árbol de mínima parsimonia. El IC fue de 0.375 y el IR de 0.416 , indicando un ajuste medio entre los datos y el árbol obtenido.

Los datos indican que todas las razas estudiadas se encuentras claramente separadas de la PAB aunque con bajos niveles de significancia. Aún así, se muestran dos grandes clusters. En uno se encuentran las razas RAF, ALT, NVA, COL, SEV, JIE, GRA, VAL, GAV, ARG, y CAN, grupo en general de pico grueso (excepto el COL y el NBA), en el otro cluster se agrupa el resto de las razas, que son de pico fino (excepto el MOR). En ambos clusters aparecen algunas razas muy agrupadas, como son el ALT y el NVA, y el COL, SEV, JIE y GRA en el primer cluster y el GAD, MAC y VEL en el segundo. El QUE, que es un típico palomo "de poca figura", aparece claramente separado de ambos clústeres, que agrupa las razas de plumaje variado (JIE, VAL y NVA tienen el plumaje azul), y se sitúa próxima a la PAB.

En la Fig. 2 se presenta el árbol de consenso estricto formado después de un centenar de réplicas bootstrap. No se manifiesta la presencia de dos clusters, como en el caso anterior, pero las mismas razas aparecen agrupadas: ALT y NVA, SEV, JIE y GRA y GAD, MAC y VEL.
Los valores obtenidos de distancias euclidianas para las características morfológicas estudiadas se muestran en el Cuadro 3. El promedio \pm desviación estándar fue de 0.90 \pm 0.15 , con valores extremos de 1.36 para el par Buchón Granadino-Paloma Bravía, y de 0.54 para los pares Buchón Valenciano-Buchón Balear y Quebrado Murciano-Colguero Argentino.

En la Fig. 3 se muestra el dendrograma obtenido a partir de las distancias euclidianas, por el método de Ward. Se observa dos clústeres aunque agrupando razas diferentes a las del cladograma. En el primer cluster, aparecen el JIE, GRA, MOR, CAN y SEV, todos ellos con el dorso recto, y el RAF, de dorso arqueado, aparece ligeramente separado. Un segundo cluster, que agrupa parte de las razas con las alas sobre la cola, podría a su vez dividirse en un grupo de buche oval, cabeza redondeada y de tamaño medio, cola media, que agruparía el GAV, VAL, ARG y ALT; y el resto, caracterizado por sus piernas rojas y verrugas escasamente desarrolladas. Ambos grupos, dentro del cluster, se diferenciarían por el grosor del pico, grueso en el primero, fino en el segundo (excepto en el NVA).

\section{Discusión}

El cladograma resultante debe ser interpretado como un árbol de grupos concernientes a las relaciones morfológicas entre las razas estudiadas y no como un árbol filogenético. Es así, que las similitudes entre grupos obtenidas en el estudio no deben usarse para agrupar las razas en un orden de tipo evolutivo.

El valor de IC obtenido (0.38) es parecido al valor reportado en un estudio en razas ovinas ( $\mathrm{IC}=0.368$ ) (Parés, 2008), que incluyó el estudio de 44 características morfológicas. No obstante, la compasión de valores de IC solo tendría sentido cuando se comparan cladogramas basados en un número 
Cuadro 1. Características morfológicas y variaciones (estados) empleados para la construcción de la matriz de similitudes morfológicas en razas de palomos buchones

\begin{tabular}{|c|c|c|c|}
\hline Característica & Estado & Característica & Estado \\
\hline A. Tamaño & $\begin{array}{l}\text { 1. Pequeño } \\
\text { 2. Medio } \\
\text { 3. Grande }\end{array}$ & $\begin{array}{l}\text { O. Grosor del } \\
\text { pico }\end{array}$ & $\begin{array}{l}\text { 1. Fino } \\
\text { 2. Medio } \\
\text { 3. Grueso }\end{array}$ \\
\hline B. Postura & $\begin{array}{l}\text { 1. Erguida } \\
\text { 2. Horizontal }\end{array}$ & $\begin{array}{l}\text { P. Longitud del } \\
\text { pico }\end{array}$ & $\begin{array}{l}\text { 1. Muy corto } \\
\text { ("apiñonado") } \\
\text { 2. Corto } \\
\text { 3. Medio } \\
\text { 4. Largo }\end{array}$ \\
\hline C. Peso vivo & $\begin{array}{l}\text { 1. Eumétrico }{ }^{1} \\
\text { 2. Subhipermétrico }\end{array}$ & $\begin{array}{l}\text { Q. Forma del } \\
\text { pico }\end{array}$ & $\begin{array}{l}\text { 1. Recto } \\
\text { 2. Ligeramente curbo } \\
\text { 3. Curbado }\end{array}$ \\
\hline $\begin{array}{l}\text { D. Color del } \\
\text { plumaje }\end{array}$ & $\begin{array}{l}\text { 1. Azules } \\
\text { 2. Cualquier plumaje }\end{array}$ & $\begin{array}{l}\text { R. Desarrollo de } \\
\text { carúnculas } \\
\text { nasales }\end{array}$ & $\begin{array}{l}\text { 1. Escaso } \\
\text { 2. Mediano } \\
\text { 3. Bastante } \\
\text { 4. No existen }\end{array}$ \\
\hline $\begin{array}{l}\text { E. Anchura del } \\
\text { pecho }\end{array}$ & $\begin{array}{l}\text { 1. Medio } \\
\text { 2. Ancho }\end{array}$ & $\begin{array}{l}\text { S. Aspecto de } \\
\text { carúnculas } \\
\text { nasales }\end{array}$ & $\begin{array}{l}\text { 1. Inexistentes } \\
\text { 2. Finas } \\
\text { 3. Desarrolladas }\end{array}$ \\
\hline $\begin{array}{l}\text { F. Perfil del } \\
\text { dorso }\end{array}$ & $\begin{array}{l}\text { 1. Recto } \\
\text { 2. Arqueado } \\
\text { 3. Ligeramente } \\
\text { arqueado }\end{array}$ & $\begin{array}{l}\text { T. Presencia de } \\
\text { verrugas }\end{array}$ & $\begin{array}{l}\text { 1. No } \\
\text { 2. Escasos } \\
\text { 3. Bastante }\end{array}$ \\
\hline $\begin{array}{l}\text { G. Longitud del } \\
\text { cuello }\end{array}$ & $\begin{array}{l}\text { 1. Corto } \\
\text { 2. Medio } \\
\text { 3. Largo }\end{array}$ & $\begin{array}{l}\text { U. Tamaño del } \\
\text { buche }\end{array}$ & $\begin{array}{l}\text { 1. Pequeño } \\
\text { 2. Escaso } \\
\text { 3. Grande }\end{array}$ \\
\hline $\begin{array}{l}\text { H. Grosor del } \\
\text { cuello }\end{array}$ & $\begin{array}{l}\text { 1. Fino } \\
\text { 2. Medio } \\
\text { 3. Grueso }\end{array}$ & $\begin{array}{l}\text { V. Forma del } \\
\text { buche }\end{array}$ & $\begin{array}{l}\text { 1. Escaso } \\
\text { 2. Oval } \\
\text { 3. En pera }\end{array}$ \\
\hline $\begin{array}{l}\text { I. Longitud de } \\
\text { las piernas }\end{array}$ & $\begin{array}{l}\text { 1. Cortas } \\
\text { 2. Medias } \\
\text { 3. Largas }\end{array}$ & $\begin{array}{l}\text { W. Colgado del } \\
\text { buche }\end{array}$ & $\begin{array}{l}\text { 1. No colgante } \\
\text { ("embebido") } \\
\text { 2. Péndulo ("buche bajo") } \\
\text { 3. Colgante ("elevado") }\end{array}$ \\
\hline $\begin{array}{l}\text { J. Grosor de las } \\
\text { piernas }\end{array}$ & $\begin{array}{l}\text { 1. Delgadas } \\
\text { 2. Medias } \\
\text { 3. Gruesas }\end{array}$ & X. "Rafe" & $\begin{array}{l}\text { 1. Presencia } \\
\text { 2. Ausencia } \\
\text { 3. Ausencia o presencia }\end{array}$ \\
\hline $\begin{array}{l}\text { K. Color de las } \\
\text { piernas }\end{array}$ & $\begin{array}{l}\text { 1. Rojo } \\
\text { 2. Morado ("pata de } \\
\text { perdiz") }\end{array}$ & $\begin{array}{l}\text { Y. Longitud de la } \\
\text { cola }\end{array}$ & $\begin{array}{l}\text { 1. Corta } \\
\text { 3. Media }\end{array}$ \\
\hline $\begin{array}{l}\text { L. Tamaño } \\
\text { relativo de la } \\
\text { cabeza }\end{array}$ & $\begin{array}{l}\text { 1. Pequeña } \\
\text { 2. Media } \\
\text { 3. Grande }\end{array}$ & $\begin{array}{l}\text { Z. Forma de la } \\
\text { cola }\end{array}$ & $\begin{array}{l}\text { 1. Llana } \\
\text { 2. Arqueada ("en hoja de } \\
\text { tilo") } \\
\text { 3. Levantada }\end{array}$ \\
\hline $\begin{array}{l}\text { M.Forma de la } \\
\text { cabeza }\end{array}$ & $\begin{array}{l}\text { 1. Redondeada } \\
\text { 2. "Almendrada" } \\
\text { 3. "Acarnerada" } \\
\text { 4. "En dado" }\end{array}$ & $\begin{array}{l}\text { AA. Alcance } \\
\text { alas-cola }\end{array}$ & $\begin{array}{l}\text { 1. Llegan a la punta } \\
\text { de la cola } \\
\text { 2. No llegan } \\
\text { 3. La superan }\end{array}$ \\
\hline $\begin{array}{l}\text { N. Aspecto de las } \\
\text { carúnculas } \\
\text { oculares }\end{array}$ & $\begin{array}{l}\text { 1. Finas } \\
\text { 2. Desarrolladas }\end{array}$ & $\begin{array}{l}\text { AB. Posición del } \\
\text { ala en reposo }\end{array}$ & $\begin{array}{l}\text { 1. Sobre la cola } \\
\text { 2. Bajo la cola }\end{array}$ \\
\hline
\end{tabular}

${ }^{1} 250-350 \mathrm{~g}$ 
Cuadro 2. Matriz de similitudes morfológicas entre razas de palom

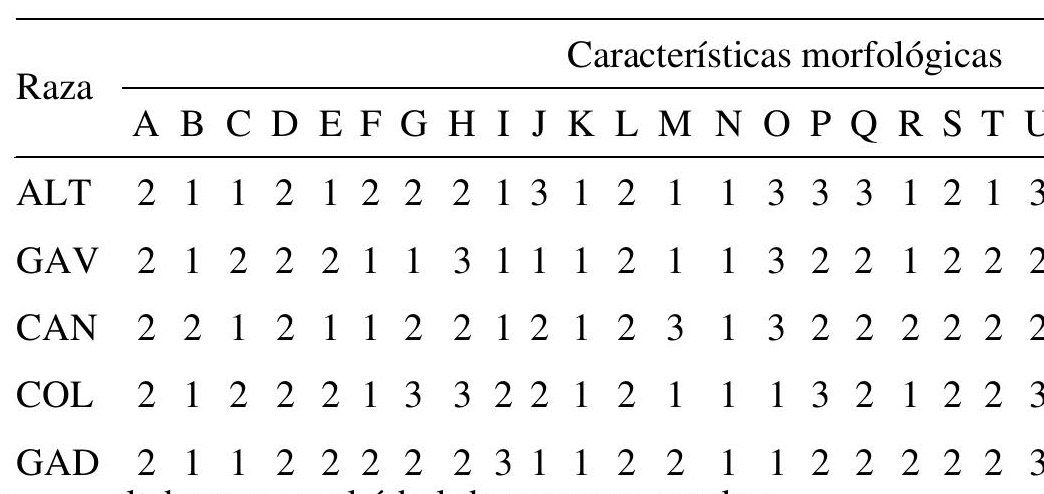

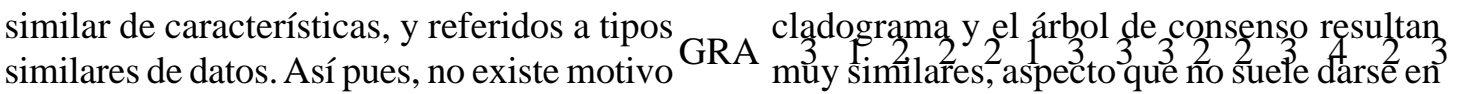
$\begin{array}{lllllll}3 & 2 & 2 & 3 & 2 & 2\end{array}$

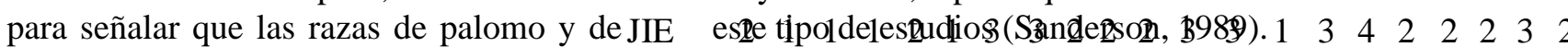
oveja puedan exhibir niveles similares de homoplasia.

SEV 2 Las razas de palomos buchones ${ }^{2}{ }^{2}$ se han $^{3}$

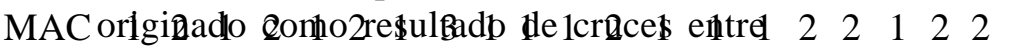
Los bajos niveles de significación en el ${ }_{\mathrm{MAT}}$ varias razas, hasta llegar ą un palomo definiárbol, indicando poca confianza, excepto en ${ }^{\mathrm{MAT}}$ do en sus características de patrón racial. Es 1

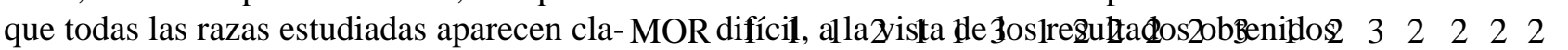
ramente separadas de la Bravía, se debe a
que las razas de buchonas están descritas $\begin{array}{llllll}\text { en este trabajo clasificar las razas en base a } \\ \text { las caracteristicas morfologicas bajo evalua- } & 3 & 1 & 2 & 2 & 2\end{array}$ como heteromorfas (Ghigi, 1908), de estruc- VAL ciðn.2Esto puede habe?sel debildo2a quellaß 2212122

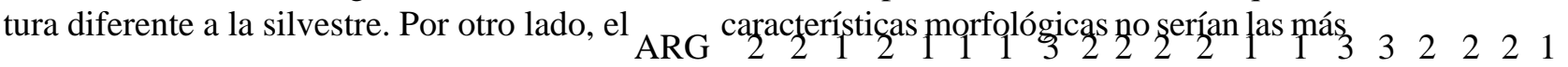


Cuadro 3. Valores obtenidos de distancias euclideanas para 28 carac

PAB ALT GAV CAN COL GAD GRA JIE

\begin{tabular}{|c|c|c|c|c|c|c|c|c|}
\hline ALT & 1.10 & & & & & & & \\
\hline GAV & 1.04 & 0.76 & & & & & & \\
\hline CAN & 0.96 & 0.76 & 0.85 & & & & & \\
\hline COL & 1.15 & 0.82 & 0.73 & 0.98 & & & & \\
\hline GAD & 1.07 & 0.89 & 0.80 & 0.85 & 0.78 & & & \\
\hline GRA & 1.36 & 1.07 & 1.00 & 0.93 & 1.02 & 0.89 & & \\
\hline JIE & 1.20 & 0.93 & 0.96 & 0.85 & 0.91 & 1.00 & 0.76 & \\
\hline SEV & 1.12 & 0.87 & 0.91 & 0.63 & 0.85 & 0.78 & 0.73 & \\
\hline MAC & 1.05 & 0.82 & 0.68 & 0.87 & 0.85 & 0.63 & 1.18 & \\
\hline MAT & 0.91 & 0.78 & 0.95 & 0.82 & 0.76 & 0.78 & 1.02 & \\
\hline MOR & 1.00 & 0.93 & 1.00 & 0.76 & 1.05 & 0.89 & 0.93 & \\
\hline QUE & 1.05 & 0.91 & 1.18 & 0.98 & 0.93 & 0.82 & 1.21 & \\
\hline VAL & 1.00 & 0.66 & 0.54 & 0.71 & 0.82 & 0.85 & 1.04 & \\
\hline ARG & 0.93 & 0.66 & 0.66 & 0.76 & 0.876 & 0.80 & 0.93 & \\
\hline VEL & 1.07 & 0.93 & 0.80 & 0.96 & 0.63 & 0.66 & 1.00 & \\
\hline CAR & 1.09 & 0.78 & 1.02 & 0.95 & 0.80 & 0.73 & 1.15 & \\
\hline NVA & B.89Inv & Wet Pe & ú(2099; & $20 .(85): 1$ & 06908 & 0.89 & 1.04 & \\
\hline RAF & 1.28 & 1.00 & 1.04 & 0.76 & 1.15 & 0.93 & 0.89 & \\
\hline
\end{tabular}




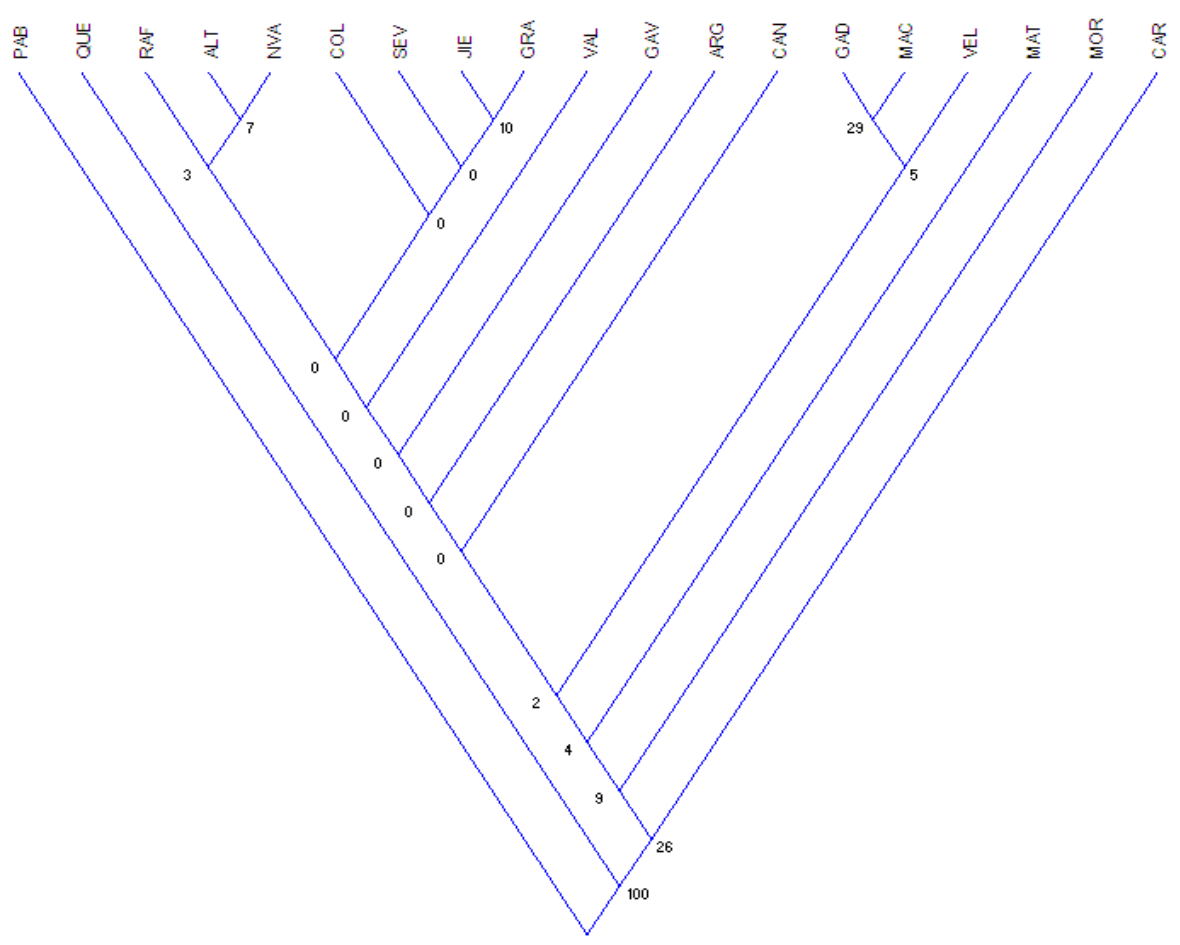

Figura 1. Cladograma resultante de la aplicación del método de parsimonia de Fitch en las características morfológicos de palomos buchones

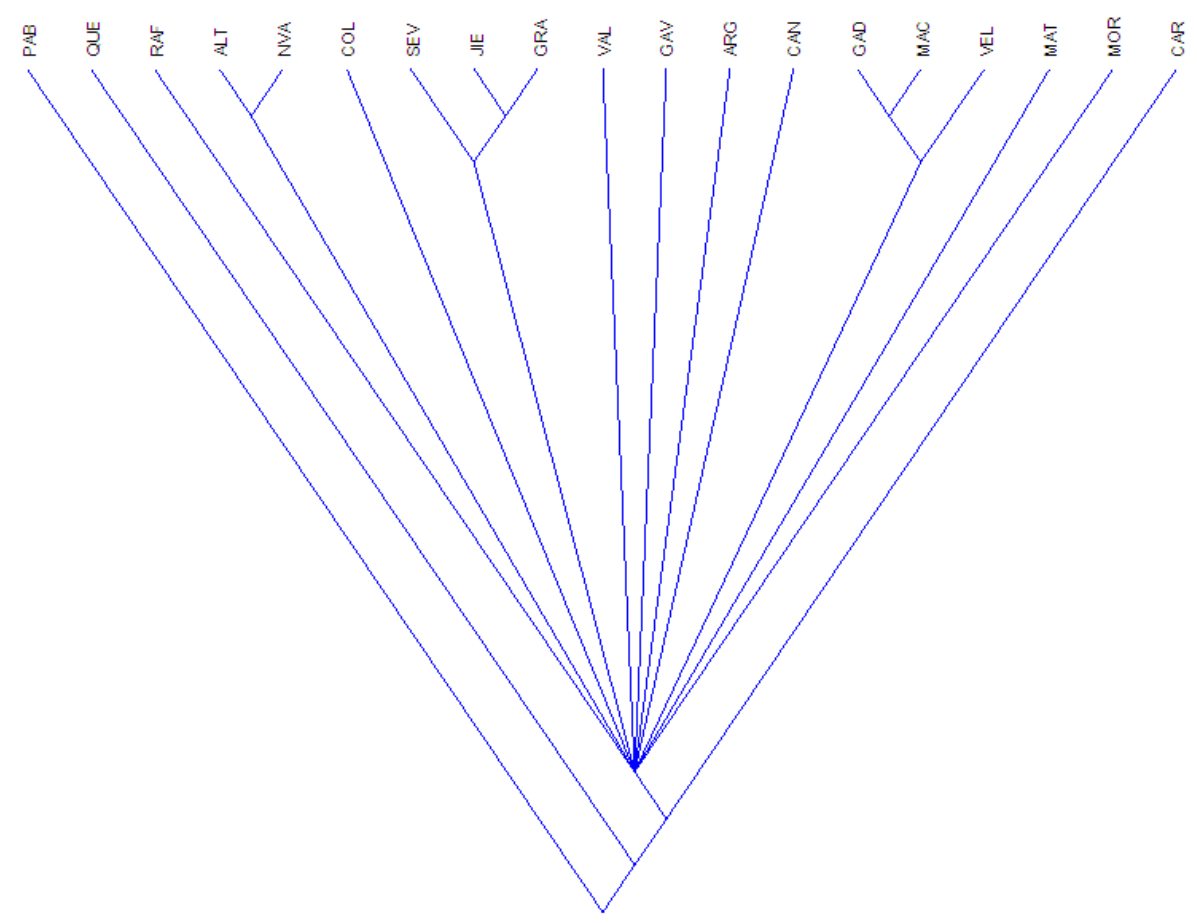

Figura 2. Árbol de consenso estricto resultante de la aplicación del método de parsimonia de Fitch en las características morfológicas de 18 razas de palomos buchones 


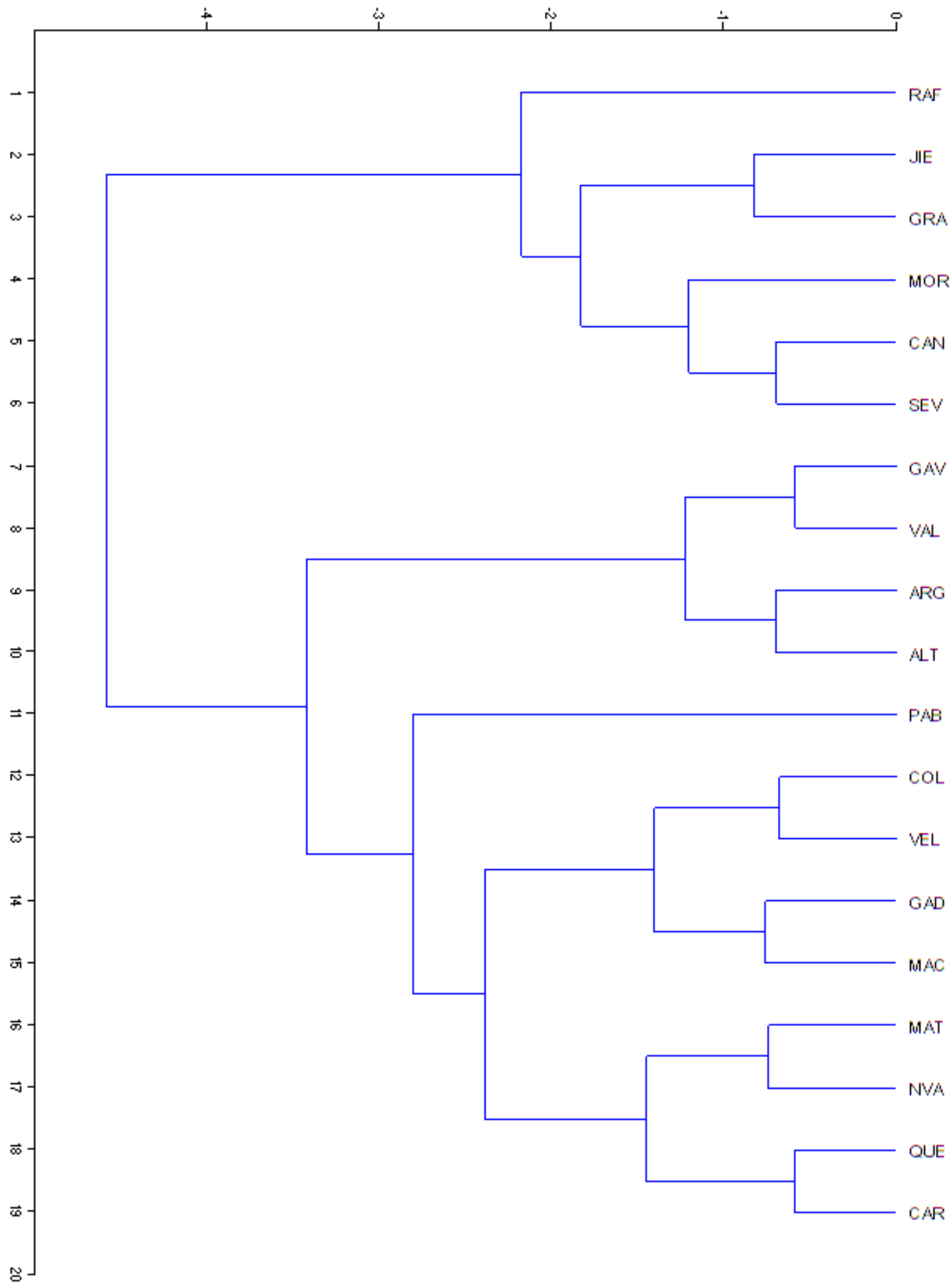

Figura 3. Dendrograma obtenido a partir de las distancias euclidianas, por el método de Ward 
discriminantes en las razas, aunque ningún estado de característica se repite en todas las razas, y quizás deberían estudiarse otros datos morfológicos, como las plumas de la albardilla (tan típica del Buchón Marteño y en el Granadino, por ejemplo), las proporciones, etc. Sin embargo, muchos de estos otros patrones raciales son específicos en las razas. Por otro lado, especialmente importante en el caso de los palomos buchones, se debería dar importancia a las características funcionales, sobretodo las relacionadas con las aptitudes de persecución, seducción y autoconservación.

Así, debería registrarse características dinámicas en el salto, en el vuelo, en el percheo, en el juego de celo y hembreo, etc. Por ejemplo, en el Buchón Gaditano, la postura en el vuelo demuestra su descendencia del Marchenero (Schille, 2005), pero esto no se muestra en los resultados obtenidos. Estas características funcionales pueden a la vez verse reflejadas tanto en el plano morfológico [por ejemplo, las características voladoras dependen de la geometría del ala, que en su aproximación más simple se define como la relación entre la longitud (envergadura) y anchura (cuerda) del ala]. Quizás los patrones raciales han ido reflejando características de orden puramente estético, relegando las de aptitud funcional.

El presente trabajo solo intenta aportar puntos de vista a las relaciones existentes entre las diferentes razas españolas de buchón, obtenidas a partir de fuentes de información morfológicas, analizadas mediante la utilización de los métodos de taxonomía numérica. Otros estudios que aportarán información de valor, además de las históricas y morfológicas, son los estudios de DNA.

\section{Literatura Citada}

1. Barba C, Lancho G, Marchal A, Valderas J. 1998. Caracterización racial del Palomo Buchón Jienense. Arch Zootec 47(178): 579 (Resumen).
2. del Hoyo J, Elliott A, Sargatal J. 1997. Handbook of the birds of the world. Vol 4. Sandgrouse to Cuckoos. Barcelona: Lynx Ed. 674 p.

3. Farris JS. 1989. The retention index and the rescaled consistency index. Cladistics 5: 417-419.

4. Ghigi A. 1908. Sulla poligenesi dei picioni domestii. Accad. Lincei 17: 271-276.

5. Gómez de Silva H, Oliveras de Ita A, Medellín RA. 2005. Columba livia. Vertebrados superiores exóticos en México: diversidad, distribución y efectos potenciales. Instituto de Ecología, Universidad Nacional Autónoma de México. Bases de datos SNIB-CONABIO. Proyecto U020. México DF.

6. Hammer $\emptyset$, Harper DAT, Ryan PD. 2001. PAST: Paleontological statistics software package for education and data analysis. Palaeontologia Electrónica 4(1) [Internet]. Disponible en: http://palaeoelectronica.org/2001_1/past/ issue1_01.html

7. Kitching IJ, Forey PL, Humphries CJ, Williams DM. 1998. Cladistics. UK: Oxford University Press. 228 p.

8. Levy WM. 1965. Encyclopedia of pigeon breeds. South Carolina: Sumter SC. Levi Publishing. 668 p.

9. Mackrott H. 1997. Palomas de raza. Barcelona: Omega. 264 p.

10. Naylor G, Kraus F. 1995. The relationship between $\mathrm{s}$ and $\mathrm{m}$ and the retention index. Syst Biol 44: 559-562.

11. Parés $P-M$. 2008. Caracterització estructural i racial de la raça ovina aranesa. Tesis de Doctorado. Barcelona: Universitat Autònoma de Barcelona. $137 \mathrm{p}$.

12. Peterson R, Mountfort G, Hollom PAD. 1980. Guía de campo de las aves de España y de Europa. Barcelona: Omega. $422 \mathrm{p}$.

13. Roelfzema J. 2007. Conservation of the gelderse slenk. Tesis de Maestría. Wageningen University [Internet]. Disponible en: http://www.abg.wur.nl/NR/ rdonlyres/39AB7BBB-2055-417C8B 7F-CCE 8 17F91CD3/54087/ Jolanda_Roelfzema_Oct2007.pdf 
14. Sanderson MJ. 1989. Confidence limits on phylogenies: the bootstrap revised. Cladistics 5: 113-129.

15. Schille H-J. 2005. Guía de las palomas de raza. Valls: Arte Avícola. 406 p.
16. Sotillo JL, Serrano V. 1985. Producción animal. I. Etnología zootécnica. Tomo II. Albacete: Artes Gráficas Flores. $296 \mathrm{p}$. 